\title{
A methodology for using Kalman filter to determine material parameters from uncertain measurements
}

\author{
Abdallah Shokry a,b,1,*, Per Ståhle ${ }^{\mathrm{a}}$ \\ ${ }^{a}$ Division of Solid Mechanics, Lund University, 22100 Lund, Sweden \\ ${ }^{b}$ Industrial Engineering Department, Fayoum University, 63514 Fayoum, Egypt
}

\begin{abstract}
A Kalman filter can be used to determine material parameters using uncertain experimental data. However, starting with inappropriate initial values for material parameters might include false local attractors or even divergence. Also, inappropriate choices of covariance errors of initial state, present state, and measurements might affect the stability of the prediction. The present method suggests a simple way to predict the parameters and the errors, required to start Kalman filter based on known parameters that are used to generate the data with different noises used as "measurement data". The method consists of two steps. First, an appropriate range of parameter values is chosen based on a graphical representation of the mean square error. Second, the Kalman filter is used based on the selected range and the suggested parameters and errors. The method of the filter significantly reduces the iteration time, and covers a wide range of initial suggested values for the parameters compared with the standard Kalman filter. When the methodology is applied to real data, very good results are obtained. Diffusion coefficient for bovine bone is chosen to be a case study in this work.
\end{abstract}

Keywords: Model, Least-Squares, Kalman filter, material parameters, diffusion in bone, uncertain measurements

\footnotetext{
*Corresponding author

Email addresses: abdallah.shokry@fayoum.edu.eg (Abdallah Shokry), per.stahle@solid.1th.se (Per Ståhle)

$1+46736925606$
} 


\section{Introduction}

The Kalman filter is an inverse method to determine variables or parameters using input data with more noise and get output data with less noise. It is firstly presented by R.E. Kalman [1] in 1960. Kalman filter has the advantages of taking the random noise for state and measurements into consideration, also it is an optimal estimator for linear models because it minimizes the mean square error between the state. In addition, it converges quickly. A more complete introduction to the Kalman filter is given by Brown [2]. The Kalman filter can be found under different updated forms that used in many different fields such as tracking objects [3-5], control systems [6, 7], and weather forecast [8,-10]. 


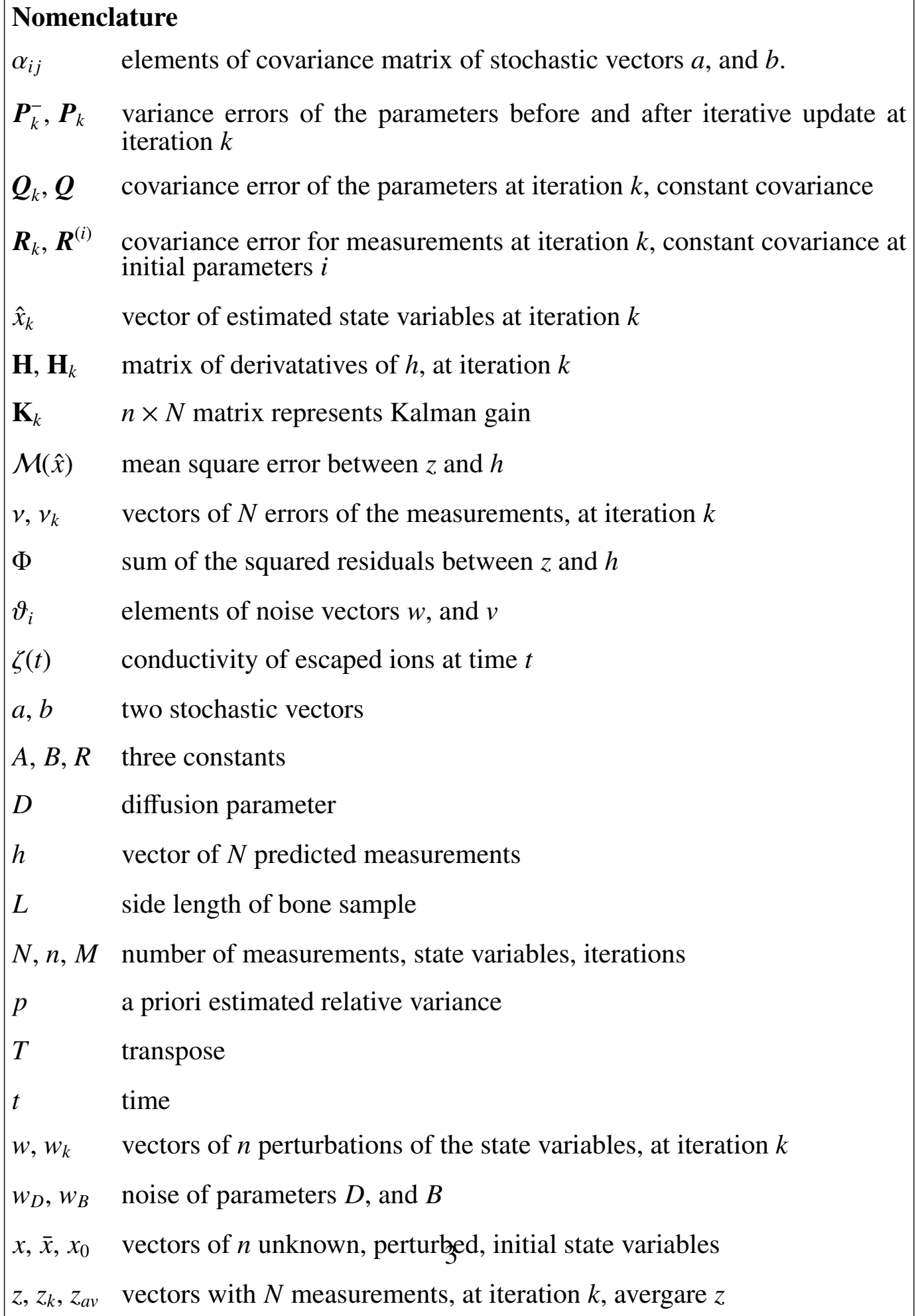


Kalman filter can be used to determine material parameters from uncertain and inaccurate measurements. Aoki et al. [11] used Kalman filter to identify Gurson's model constant. They found that the accuracy of parameters prediction is affected by both specimen geometry and measurement type, and the shape of the tested specimen affects the convergence of the parameters. Also, they noticed that the rate of convergence can be improved by combining measurements of two different specimens in shape. The identification of Gurson-Tvergaard material model parameters via Kalman filtering technique is studied by Corigliano et al. [12]. They stated that the estimated values of the parameters are in well agreement with those obtained in previous work, but the initial suggested values for the seeking parameters affects the estimated parameters.

Nakamura et al. [13] implemented Kalman filter to determine elastic-plastic anisotropic parameters for thin materials using instrumented indentation. They observed that the initial chosen values for the parameters converged to a specific small area, but not to one point. Also, based on the convergence intensity, the parameters are determined. The same findings are obtained by using Kalman filter to determine the nonlinear properties of thermal sprayed ceramic coatings [14]. Bolzon et al. [15] used Kalman filter to identify parameters of a cohesive crack model. They reported that almost a linear correlation between convergent parameters is found, and the reason for the multiple local minimum might be related to using the linear Kalman filter for non-linear model.

Vaddadi et al. [16] used Kalman filter to determine critical moisture diffusion parameters for a fiber reinforced composite. They estimated the parameters from the intensity of the convergence, which found to be in consistent with known values. Another study made by Vaddadi et al. [17] to determine hygrothermal properties in fiber reinforced composite using Kalman filter. The parameters are extracted by reading the intensity of convergence plot.

Kalman filter is an efficient way to filter noisy experimental data for determination of material parameters. However, the initial suggested parameters required for Kalman filter should be chosen carefully, to avoid false local attractor. Also, the covariance error for the parameters noise almost assumed to be zero, which slow the rate of convergence and might lead to more than one intensity area for the predicted parameters.

In this study, a methodology will be applied for using Kalman filter to determine material parameters using uncertain measurements. The methodology starts by a way based on the mean square error to choose appropriate initial suggested parameters required for Kalman filter, and followed by a suggested way to choose the covariance errors for both state and measurements. The determination of dif- 
fusion coefficients in bovine bone for generated data with different noises scatter from known parameters will be applied as a case study. A real measurements will be used also.

\section{Methods}

\subsection{The Model}

Assume that an experiment resulted in $N$ measurements obtained at different times, locations, temperatures etc. These are collected in a vector, $z$, with $N$ measurements. The experimental data may be obtained at different known times, locations, temperatures etc. Measurements and all other data are available a priori.

In an attempt to predict the measurements a model, $h=h(x)$ is used, with $h$ being a vector of $N$ predictions of observations. Further, $x$ is a vector of $n$ unknown parameters defining the model based on variables such as position, temperature, time, etc. The unknown model parameters may describe the state of the system regarding, material, geometry or similar. In the present study, $x$ is limited to parameters describing the material.

Measurements always include systematic and non-systematic errors due to instrumentation, indirect observations, gauges sensitive, irrelevant external influence, and similar. Material parameter is sought but the experimental method may require a state parameters to be determined as well. Further, material parameters contain non-systematic errors due to thermal fluctuations, unstable structural configurations such as mobile dislocations, impurities, inclusions, unstable chemical composition, etc. Also inevitably, there is a difference between model and reality while a model never gives an exact description of the physical processes. Under ideal conditions the model would perfect in the sense that $z=h(x)$. Here, only non-systematic errors or noise is considered. The model is defined for measurement $i$ as

$z=h(\bar{x})+v$,

where $v$ is a vector with $N$ errors due to inaccurate measurements $z$. The instant parameter $\bar{x}$ corresponding to the individual measurement $i$ includes noise according to

$\bar{x}=x+w$,

where $w$ is a vector with $n$ errors caused by the parameter deviations. The elements of $v$ and $w$ are assumed to be uncorrelated. All elements of $w$ and $v$ are supposed 
to be random, having the same respective stochastic distribution and for both a vanishing mean value is expected, cf. [2].

Assuming that a set of parameters $\hat{x}_{k}$ is an estimate in the neighborhood of $x$, an improved estimate $x_{k+1}$ may be obtained by using linearized using a Taylor series which gives

$h\left(\hat{x}_{k+1}\right) \approx h\left(\hat{x}_{k}\right)+\mathbf{H}\left(\hat{x}_{k}\right)\left(x_{o}-\hat{x}_{k}\right)$,

when quadratic and higher order terms of $x$ are neglected. On matrix form involved variables are

$h(x)=\left[\begin{array}{c}h^{(1)} \\ \vdots \\ h^{(N)}\end{array}\right], \mathbf{H}(x)=\left[\begin{array}{ccc}\frac{\partial h^{(1)}}{\partial x_{1}} & \cdots & \frac{\partial h^{(1)}}{\partial x_{n}} \\ \vdots & \ddots & \vdots \\ \frac{\partial h^{(N)}}{\partial x_{1}} & \cdots & \frac{\partial h^{(N)}}{\partial x_{n}}\end{array}\right], x=\left[\begin{array}{c}x_{o} \\ \vdots \\ x_{n}\end{array}\right]$.

Here, $\mathbf{H}$ is an $N \times n$ a Jacobian matrix.

\subsection{Least-Squares}

The system is supposed to be overdetermined, meaning that the number of measurements $N$ exceeds the number of unknown parameters $n$. The best fit in the least-squares sense minimizes the sum of squared residuals with a residual being the difference between an observations $z$ and the predictions $h(x)$. The unknown $x$ is obtained in a series of iterative improvements of the approximation $x \approx \hat{x}_{k}$, where $k$ is the iteration number. The initial parameters $x_{0}$ may be an educated guess based on previous measurements, data from resembling materials or other similar expectations.

Solutions for non-linear systems (see Appendix A) may be obtained iteratively. As an example, the Newton-Raphson method applied to these solutions gives the following recursive scheme,

$\hat{x}_{k+1}=\hat{x}_{k}+\left(\mathbf{H}_{k}^{\mathrm{T}} \mathbf{H}_{k}\right)^{-1} \mathbf{H}_{k}^{\mathrm{T}}\left\{z-h\left(\hat{x}_{k}\right)\right\}$,

where $\mathbf{H}_{k}$ is an $N \times n$ matrix and a function of $\hat{x}_{k}$. If convergence is reached, a local minimum of the sum of squared residuals has been found. To find global minimum, additional steps have to be taken. The drawback of the method is that convergence is not necessarily reached and is less likely if the a priori information of $x$ is vague, unreliable or even misleading. This is especially accentuated when the measurements are noisy. Further, all data have to be present a priori. Modifications have been developed that allow an incremental treatment, which may be useful if data is continuously added (cf. [18]). 


\subsection{Kalman Filter}

The method of least-squares does not take the properties of the noises $v$ and $w$ as regards expected mean value and distribution into account. Opposed to that, the Kalman filter is developed to use information about the noise a priori or as the measurements are assembled. The filter is an improvement of the least-squares method as it recursively optimizes the unknown model parameters in search for least sum of squared errors. The method is developed with the particular endeavor to effectively handle noisy input data [2]. It is also operating incrementally so that new may be added as they appear in during ongoing measurements without loss of accuracy. However, in the present study all measurements are supposed to be available when the optimization is initiated. The Kalman method is generally assumed to be an effective method to filter noisy data combined with a high convergence rate. The derived algorithm is taking the character of the noise into consideration. The expected vanishing mean values for $v$ and $w$ are explicitly utilized.

Assuming that the measured data is the predicted data based on the optimum material parameters with the addition of noise, as given by Eq. (11) it is here assumed that the noise $v$ a distribution with a zero mean value.

Initially a set of parameters $x_{0}$ is selected based on a priori information from other measurements under same or similar conditions or otherwise known data. From this, the parameters are iteratively updated using an algorithm on the same form as the least-square algorithm (cf. Eq. 5) as follows

$\hat{x}_{k+1}=\hat{x}_{k}+\mathbf{K}_{k}\left\{z_{k}-h\left(\hat{x}_{k}\right)\right\} \quad$ for $\quad k=0,1,2, \ldots M$,

where $\mathbf{K}_{k}$ is an $n \times N$ matrix denoted the Kalman gain, and $M$ is the number of iterations. In Eq. (6). Normally the Kalman algorithm operates on single measurements one by one so that the Kalman gain is updated for every new measurements. This may be necessary for interactive processes where the action requires knowledge of the instantaneous state of the system. In the present study, iterations are simultaneously utilizing all measurements. The derivation of the Kalman gain (see Appendix B is based on measurements added recursively as in the original form of the filter.

The optimal $\mathbf{K}_{k}$ that minimizes the mean square error is given by

$\mathbf{K}_{k}=\boldsymbol{P}_{k}^{-} \mathbf{H}_{k}^{\mathrm{T}}\left(\mathbf{H}_{k} \boldsymbol{P}_{k}^{-} \mathbf{H}_{k}^{\mathrm{T}}+\boldsymbol{R}_{k}\right)^{-1}$.

where $\boldsymbol{P}_{k}^{-}$is the variance of the errors of the parameters before the iterative update, and $R_{k}$ is $N \times N$ matrix introduces the covariance error of the measurements that computed as 
$\boldsymbol{R}_{k}=\operatorname{Var}\left(v_{k}\right)$.

The $\boldsymbol{P}_{k+1}^{-}$is expressed as

$\boldsymbol{P}_{k+1}^{-}=\left(\boldsymbol{I}-\mathbf{K}_{k} \mathbf{H}_{k}\right) \boldsymbol{P}_{k}^{-}+\boldsymbol{Q}_{k}$,

where $\boldsymbol{Q}_{k}$ is an $n \times n$ matrix represents the covariance errors for the state parameters that is computed as

$\boldsymbol{Q}_{k}=2 \operatorname{Var}\left(w_{k}\right)$,

The derivation of $\mathbf{K}_{k}, \boldsymbol{P}_{k}^{-}, \boldsymbol{R}_{k}$, and $\boldsymbol{Q}_{k}$ are given in details in Appendix B.

The algorithm involves recursive use of the Eqs. (6), (7) and (9). The measurements considered in each recursive cycle may be everything from a single measurement to all measurements. For non-linear problems, each cycle may be repeated until convergent results are obtained. As an alternative, the entire recursive scheme may be restarted and the resulting parameters $x_{n}, \boldsymbol{P}_{n}^{-}$, from previous application of the scheme are used as initial parameters. Also $\boldsymbol{Q}_{k}$ and $\boldsymbol{R}_{k}$ may be adjusted based on the improved information that is obtained. In the present study, all measurements are placed in a single set with $N$ measurements, meaning that $z_{k}=z$ is a constant vector with $N$ elements. The number of recursive cycles is $M$ and $k=1,2, \ldots, M$ where $M$ is prescribed or conditional. The recycling is performed to achieve a converged result for a non-linear problem. In each cycle, $x_{k}$, $\mathbf{H}_{k}, \boldsymbol{P}_{k}$ and therefore $\mathbf{K}_{k}$ is updated.

By putting $\boldsymbol{R}_{k}=R \boldsymbol{I}$ in Eq. (7) and then taking the limiting result as $R \rightarrow 0$ one obtains $\mathbf{K}_{k}=\mathbf{H}_{k}^{\mathrm{T}}\left(\mathbf{H}_{k} \mathbf{H}_{k}^{\mathrm{T}}\right)^{-1}=\left(\mathbf{H}_{k}^{\mathrm{T}} \mathbf{H}_{k}\right)^{-1} \mathbf{H}_{k}^{\mathrm{T}}$. After inserting this into Eq. (6) it is readily seen that the result is identical to that of the non-linear least square method, cf. Eq. (5). The result is independent of $\boldsymbol{P}_{k}^{-}$and consequently also independent of $\boldsymbol{Q}_{k}$.

\section{Methodology}

In [11, 13, 17], the $\boldsymbol{R}_{k}$ was chosen to be a small percentage of the measured data, and the $\boldsymbol{Q}_{k}$ value was chosen to be zero. In a recent study, the $\boldsymbol{R}_{k}$ value was chosen as the difference between the measured data and a predicted data a round the measured data, and the $\boldsymbol{Q}_{k}$ was chosen to be unity [19].

To use the Kalman filter the parameters $\boldsymbol{Q}_{k}$ and $\boldsymbol{R}_{k}$, and initial values for $\hat{x}_{0}$ and $\boldsymbol{P}_{0}^{-}$have to be defined. In the following, different strategies for choosing these values is described. 
For common usage of the Kalman filter, the choice would be $\boldsymbol{R}_{k}=\operatorname{Var}\left(v_{k}\right)$ and $\boldsymbol{Q}_{k}=2 \operatorname{Var}\left(w_{k}\right)$ according to Eqs. (8) and (10). These variations are assumed to be known a priori, at least approximately. The information may be based on expectation or derived from the present measurements, using a large variety of hypotheses. When the method is used recursively, the indices $\mathrm{k}$ allow for using $\boldsymbol{Q}_{k}$ or $\boldsymbol{R}_{k}$ or both as functions of time, position, etc.

The Kalman gain, given by Eq. (7), with the selected $\boldsymbol{Q}_{k}$ or $\boldsymbol{R}_{k}$, minimizes the squared error of the estimate $\hat{x}_{k}$ of $x$. A condition for the derivation is that $h(x)$ is a linear function of the parameters $x$. In the present study, $h(x)$ is a non-linear function of $x$. The aim is to formulate a strategy for selecting the free parameters $\boldsymbol{R}_{k}$ and $\boldsymbol{Q}_{k}$, not necessarily according to Eqs. (8) and (10), so that the square of the error

$\mathcal{M}(x)=\frac{1}{N} \operatorname{Var}(z-h(x))$,

is minimized. The study does not attempt to be exhaustive and the conclusions are empirical and based on a case study. The selection of method is primarily based on convergence rate. With a wide range of starting values, occasionally the estimate converges outside the range of interest and there is also the risk of failure in producing converging results at all. These, disadvantages are also considered in the selection of a suitable procedure for selecting $\boldsymbol{R}_{k}$ and $\boldsymbol{Q}_{k}$.

In the first part of the study, the initial parameters $\hat{x}_{0}$ are selected to cover a several orders of magnitude wide range of values. Under normal circumstances, this cannot be done for non-linear phenomena or realistic geometries or anything else for which an analytical solution cannot be found, which may be the general case. When the predictions are based on non-linear numerical calculations of field problems, e.g., using lengthy finite element analyses, usually only a few initial parameters $\hat{x}_{0}$ can be considered. Here however, a wide range of initial parameters is examined as regards the mean square error $\mathcal{M}\left(\hat{x}_{0}\right)$. The aim is to obtain an overall picture of the possibilities of fast convergence or difficulties because of present local minima, sadle points etc.

The second part is, the using of a suggested method and comparing the resulting convergence rate, the ability of producing convergent results, and the percentage of convergent results from a range of the initial parameters with three additional methods. All four methods are described in subsection 3.1.

\subsection{Selecting $\boldsymbol{Q}_{k}$ and $\boldsymbol{R}$}

In the present study, the results of constant 
$\boldsymbol{Q}_{k}=2 p^{2} x x^{\mathrm{T}}$ including $\boldsymbol{Q}_{k}=0$,

are evaluated. The parameter $p$ is an a priori estimated relative variance.

The relative variance $p$ of the state parameters is a quantity that possibly can be guessed with more or less accuracy. Since the real state parameters $x$ are not known a priori constant $\boldsymbol{Q}_{k}$ may be either too big or too small depending on how accurate initial guess of $x_{0}$ is. However, since the knowledge of the state variables increases as the iterations proceed, to stick a constant $\boldsymbol{Q}_{k}$ may unnecessarily slow down the convergence rate. As the iterations proceed, the estimate $x_{k}$ is improved and a better estimate for $\boldsymbol{Q}_{k}$ can be used. Here, an updated $\boldsymbol{Q}_{k}$ is also evaluated. With the variation of $x$ being $w_{k}=p x$, the unknown $x$ is here assumed to best approximated with $x_{k}$. The updated value given by Eq. (10) then becomes

$\boldsymbol{Q}_{k}=2 p^{2} \operatorname{Var}\left(x_{k}\right)$.

The $\boldsymbol{R}_{k}$ taken as

$\boldsymbol{R}^{(i)}=v^{(i)} I, i=1,2, \ldots .1681$,

which is assumed to be a reasonable approximation of Eq. (8), where measurement noise $v^{(i)}$ is given by

$\left.v^{(i)}=\max \left|\operatorname{Var}\left(z_{N}-h_{N}\left(\hat{x}_{0}^{(i)}\right)\right)\right|\right]$

where max denoted to the maximum value, and $i$ is the number of initial parameters.

In the suggested method, the $\boldsymbol{Q}_{k}$ and $\boldsymbol{R}^{(i)}$ are chosen for large values based on the evaluation according to Eqs. (7) and (9), cf. section 5 for explanation. There are additional two Kalman filter methods, one method uses $p=0$ in Eq. (12), and the another method uses $p=0.01$ that gives the smallest standard deviation and largest percentage of convergence among different values for $p$, the mean values are closest to each other (see Fig. 4). These two methods use $\boldsymbol{R}_{k}$ as

$\boldsymbol{R}=\left[\max \left|\operatorname{Var}\left(z_{N}-h_{N}(x)\right)\right|\right] I$,

so, the $\boldsymbol{R}$ is the largest squared element for the variance of the difference between measured data, $z$, and predicted data with noise. Here, $\boldsymbol{Q}$ and $\boldsymbol{R}$ without $k$ index denote constant covariances during iterations.

The fourth method is the non-linear least square method, which is obtained by letting $\boldsymbol{R}_{k} \rightarrow 0$ as it is described in subsection 2.3 . 


\subsection{The Initial Predicted Parameters Error $\boldsymbol{P}_{0}^{-}$}

The initial predicted parameters error $\boldsymbol{P}_{0}^{-}$is an $n \times n$ matrix that contains the expected values for the errors between the unknown parameters $x_{k}$ and the initial predicted parameters $\hat{x}_{0}$ before the first iteration (see Appendix B). $\boldsymbol{P}_{0}^{-}$is chosen as

$\boldsymbol{P}_{0}^{-}=\operatorname{Var}\left(\hat{x}_{0}^{(\max )}-\hat{x}_{0}^{(\min )}\right)$.

where $x_{0}^{(\max )}$ and $x_{0}^{(\min )}$ are the two vectors that contain maximum and minimum of the selected initial values for the parameters. The choice for $\boldsymbol{P}_{0}^{-}$is the same in all cases in the present study. Only one large initial value is tested since the $\boldsymbol{P}_{0}^{-}$ introduces the variance between the seeking parameters and the initial parameters.

\subsection{Summary of Methods}

Table 1: Methods used

\begin{tabular}{|c|c|c|c|c|}
\hline Method & $\mathrm{Q}$ & $\mathrm{R}$ & $\mathrm{P}$ & D.N\% \\
\hline $\begin{array}{c}\text { Kalman filter } 1 \\
\text { (suggested method) }\end{array}$ & $\boldsymbol{Q}=\boldsymbol{P}_{0}$ as in Eq. 23 & Eq. 26. & Eq. 17 & 92.09 \\
\hline Kalman filter 2 & Eq. 112, with $p=0$ & Eq. 16. & Eq. 17 & 80.90 \\
\hline Kalman filter 3 & Eq. (12) with $p=0.01$ & Eq. (16) & Eq. $17 \overline{17}$ & 80.19 \\
\hline Non-linear least square & - & 0 & - & 40.93 \\
\hline
\end{tabular}

\section{Case Studies}

The determination of the diffusion coefficient of mammal bone using uncertain data is chosen to be a case study. Diffusion has recently been suggested to play an important role in transporting substances from the inner boundaries to the outer boundaries of bone. Therefore, knowing the diffusion coefficients in human bone are important to give required information for design of individual exercise programs that maximizes bone remodeling and bone healing.

In the present study, the proposed four methods are applied to several simulated cases of generated data and the most effective method is applied to a case of real experimental data. The real experiment measures the amount of ions that leaves bovine bone samples that were put into a container with distilled water. During elapsing time the conductivity, $\zeta(t)$, of the water increases in proportion 
to the ionic concentration. The experiment is reported in Lindberg et al. [19]. Cubic bone samples with the side length $L=10.1 \mathrm{~mm}$ from a bovine long bone were used. The concentration were measured using a SevenEasy S30 conductivity meter from Mettler Toledo with an accuracy of $\pm 0.5 \%$ of the measured value. Further details regarding the experiment is found in [19].

The following model is suggested for the conductivity as a function of time, $t$,

$\zeta(t)=A-B \sum_{m=1}^{M} \frac{8}{\pi^{2}(2 m-1)^{2}} \exp \left\{-(2 m-1)^{2} \pi^{2} D \frac{t}{L^{2}}\right\}$,

where $D$ is the diffusion constant, and $A$ and $B$ are unknown constants. The model is based on Fick's law using relevant boundary conditions.

The constants $A$ and $B$ provide the relation between the concentration in the bone sample and the conductivity in the distilled water. Putting $t=0$ gives

$A=\zeta(0)+B$

where $\zeta(0)$ taken to be the conductivity measured at $t=0$. By using $h(t)=$ $\zeta(t)-\zeta(0)$ as the measured quantity, the following model is obtained,

$h(t)=B\left(1-\sum_{m=1}^{M} \frac{8}{\pi^{2}(2 m-1)^{2}} \exp \left\{-(2 m-1)^{2} \pi^{2} D \frac{t}{L^{2}}\right\}\right)$,

The two remaining constants $D$ and $B$ is determined using the Kalman filter. More details are found in Lindberg et al. [19].

To the experiment is added a Monte Carlo set of 250 fictive measurements where generated data is used as measurements. The measured data is generated using the exact model Eqs. (1) and (20) to compute the measurement vector $z$. To provide realistic conditions, a variation is added to the state parameters $D$ and $B$ and to the measurements. To this end, a random noise of $5 \%$, i.e. $\left|w_{D}\right|<0.05 D$ and $\left|w_{B}\right|<0.05 B$, is added. In the same way, noises $v$ of $10 \%, 50 \%$ and $100 \%$ are included in the generated measurements $z$, i.e. $z=h(t)+v$, where $v=q \frac{1}{N} \sum h\left(t_{i}\right)$ and $q$ equals $0.1,0.5$ and 1. Summation is performed for the 24 different times of measurement. Further, the $w_{D}, w_{B}$ and $v$ are uncorrelated and the probability density is constant within the limits of the respective noise. Also the noises for each measurements are uncorrelated. Thus,

$\operatorname{Cov}\left(\vartheta_{i}, \vartheta_{j}\right)=\left\{\begin{array}{ccc}\vartheta_{i}^{2} & \text { if } & i=j \\ 0 & \text { if } & i \neq j\end{array}\right.$ 
where $\vartheta_{i}$ and $\vartheta_{j}$ represents the elements of each noise vector $w_{D}, w_{B}$ and $v$ at individual measurements $i$ and $j$.

As observed from Eq. 20, the only available time unit is provided by $L^{2} / D$. A typical experiment lasts for around 11.7 time units, i.e., $0 \leq t \leq 11.7 L^{2} / D$ with generated measurements taken in time intervals of around $0.0032 L^{2} / D$.

In an attempt to obtain less non-linear formulation of Eq. (20), a semi-linear model that is obtained after simplification and taking log of both sides of Eq. (20) one obtains

$h^{*}(t)=B^{*}+f(D, t)$,

where

$h^{*}(t)=\log \{h(t)\}, B^{*}=\log (B)$,

and

$f(D, t)=\log \left(1-\sum_{m=1}^{M} \frac{8}{\pi^{2}(2 m-1)^{2}} \exp \left\{-(2 m-1)^{2} \pi^{2} D \frac{t}{L^{2}}\right\}\right)$

which makes $h^{*}(t)$, a linear function of $B^{*}$ while $f(D, t)$ is known to be rather small. Note that $\log \left(1-e^{-x}\right) \rightarrow e^{-x}$ as $x \rightarrow \infty$ and therefore $f(D, t)$ decays exponentially for large with increasing time which makes the linearization with respect to $D$ undoable.

\section{Results and Discussions}

The accuracy of the series in Eq. (20) was studied in this work to reduce the computational time, it showed that the number of terms $m$ may be chosen to be around 200 terms to obtain an accuracy of $99.9 \%$ in the middle of the sample at $t=0$. Already at the second measurement at $t=0.003 L^{2} / D$ only four terms are required to obtain the same accuracy. For systems with large amounts of data, a strategy for the selection of the number of terms in the series could save considerable computation time. However, here this is not the primary focus and the the calculations where not very much time consuming, which is why all calculations where made using $M=200$ terms.

Measurements from known $D$ and $B$ using $w_{D}=0.1 D, w_{B}=0.1 B$, and $v=0.1 z_{a v}$ where $z_{a v}$ is the average of all generated measurements i.e $z_{a v}=\frac{1}{N} \sum z_{i}$. Then the Kalman filer was used to obtain approximations of $D$ and $B$. This was 
done first for the non-linear model Eq. (20), and then for the partly linearized model Eq. (22). To compare the accuracy in finding the least square error of the predicted measurements based on different state parameters found using model Eq. (20) and model Eq. (22) respectively, the least square error $\mathcal{M}(x)$ was calculated using Eq. (11). The non-linear model gave $\mathcal{M}(x)=15 \%$ while the partly linearized model gave $30 \%$. For the reason of this, the partly linearized model is given up and the model Eq. (20) is used in the continued analysis.

Assuming that the information of the parameters $D$ and $B$ is uncertain, the Kalman filtering has to converge from initial values that are several orders of magnitudes different from the true values. To explore what this means, the mean square error is calculated for a very wide range of initial values. Mean square error here refers to the error of calculated estimates of the initial guesses $D_{0}$ and $B_{0}$ directly compared with the true values of $D$ and $B$. No iterations are made. To cover a large variety of initial values a mesh 101 values for $D$ and for each 101 values for $B$ are used. The mesh covers values of $D_{0}$ ranging from $0.001 D$ to $100 D$ and values of $B_{0}$ from $0.01 B$ to $100 B$. Fig. 1 shows the mean square error for the initial values of $D_{0}$ and $B_{0}$ for the constructed mesh.

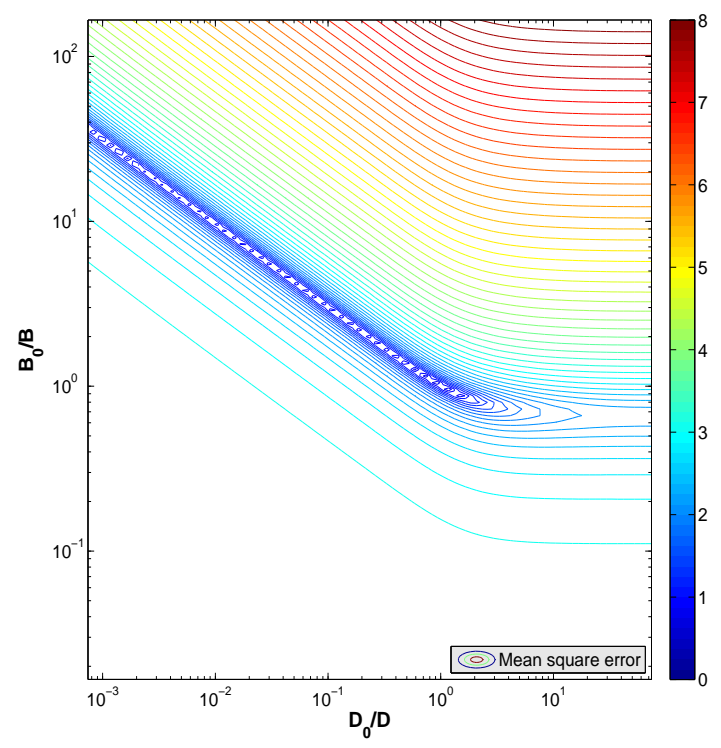

Fig. 1. The mean square error of the conductivity predictions for different initial values of the relative initial parameter values $D_{0} / D$ and $B_{0} / B$. The noises are in the range $\pm 5 \%$ for parameters and $\pm 10 \%$ for measurements. 
Regions with small gradients are found along a line for which $D_{0} / D$ approximately equals $\left(B_{0} / B\right)^{-2}$. The several local minima along this trajectory correspond to the resolution of the grid and are merely graphical anomalities. Also in the region of small $D_{0}$ and small $B_{0}$ the gradient is very small. This makes the convergence rate of any gradient driven algorithm small. However, no local minimum seems to be present and convergence should be possible in entire range of initial values even if the convergence may be very slow in the above describe regions. A clear minimum mean square error is found around the close to $D$ and $B$. The result is strongly influenced by the rapid changes due to the exponential behavior of Eq. (20).

For the study of the Kalman filter measurements $z$ are generated for 250 measurements in the time interval $0 \leq t \leq 11.7 L^{2} / D$. In this study, $D$ and $B$ are known parameters, but in a real case they are not known but believed to be in the neighborhood of the a priori guess. As it is suggested by Fig. 1, a very large variation of convergence rates are anticipated.

The Kalman filter is studied by gathering 10 generated experiments together, each experiment has 25 measurements, and each measurement has random noises of $\pm 5 \%$ for parameters and $\pm 10 \%, \pm 50 \%$, and $\pm 100 \%$ for measurements, which constructs a measurement vector $z_{i}, \mathrm{i}=1,2.3 \ldots . ., 250$. The initial selected state variables are chosen from $0.1 D$ to $10 D$ for $D_{0}$ and from $0.1 B$ to $10 B$ for $B_{0}$.

The initial parameters error $\boldsymbol{P}_{0}^{-}$is selected according to Eq. (17) as follows

$$
\boldsymbol{P}_{0}^{-}=\left[\begin{array}{cc}
9.9^{2} D_{0}^{2} & 0 \\
0 & 9.9^{2} B_{0}^{2}
\end{array}\right],
$$

The $\boldsymbol{P}_{k}^{-}$is updated using Eq. (9).

\subsection{Different $\boldsymbol{R}$ and $\boldsymbol{Q}_{k}$}

First $\boldsymbol{R}^{(i)}$ values are calculated as in Eq. (14). So, $\boldsymbol{R}^{(i)}$ is the squared largest element of the variance encountered so far, i.e. so far means before iteration $k$.

The effect of $\boldsymbol{Q}_{k}$ is studied by using,

$\boldsymbol{Q}_{k}=\left[\begin{array}{cc}\left(D_{k}-D_{0}\right)^{2} & 0 \\ 0 & \left(B_{k}-B_{0}\right)^{2}\end{array}\right]$.

This means that $\boldsymbol{Q}_{k}$ increases as the iterations proceed. The expected effect is that the convergence rate increases with increasing $\boldsymbol{Q}_{k}$. Which is selected to speed up the convergence rate. 
Fig. 2(a) shows a color plot of the obtained values for $D_{1} / D$, here 1 is number of iterations. The markers $(x)$ that are included show the obtained values of $D_{1} / D$ and $B_{1} / B$ using Kalman filter, for $41 \times 41$ initial values $D_{0} / D$ and $B_{0} / B$. The obtained $\left(D_{1} / D, B_{1} / B\right)$ outside the selected range are excluded from convergence plot to make it in the same range as the color plot. The white areas in the figure give $21.5 \%$ of the obtained $D_{1} / D$ that found to have negative values with no physical meaning. Consequently, this leads to divergent result in following iteration since the exponential term in the diffusion model would have large positive values. A large step for Kalman gain is $\mathbf{K}_{k}$ seems to be the reason, which can be forced to be small by assuming $\boldsymbol{R}$ large, but the rate of convergence would be slow.
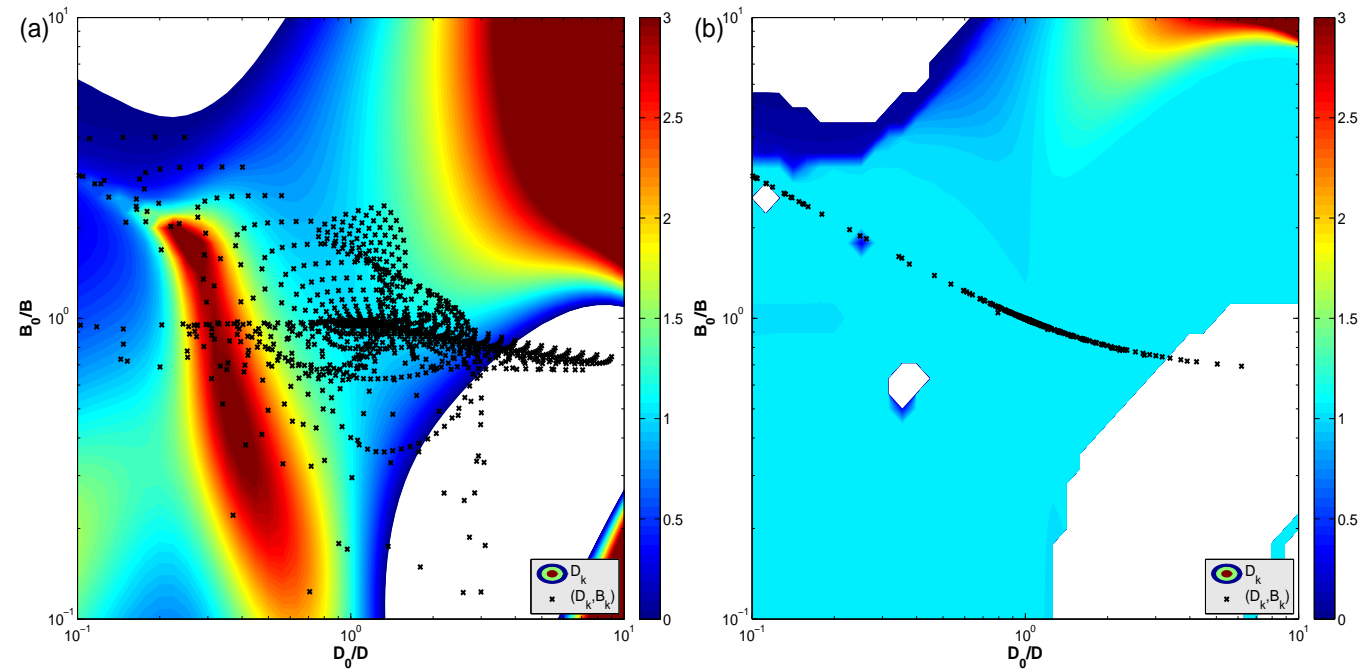

Fig. 2. Color plot of $D_{k}$ as a function of $D_{0}$ and $B_{0}$. The markers $(\times)$ show the position of the resulting $D_{k}$ and $B_{k}$ for the 1681 initial starting points in the range. (a) $k=1$ iteration, and (b) $k=20$ iterations. Noises are $\pm 5 \%$ for the parameters and $\pm 10 \%$ for the measurements.

Fig. 2(b) shows the color and convergence plot after 20 iterations. It can be seen that using larger values for $\boldsymbol{Q}_{k}$ increases the rate of convergence but the divergent cases increased from $21.5 \%$ to $22.4 \%$.

The effect of $\boldsymbol{R}$ values on the convergent $D_{1} / D$ is shown in Fig. 3(a). The figure shows that the percentage of the number of convergent $D_{1} / D$ over the total 
number of $D_{1} / D$ almost around $80 \%$ as the $\boldsymbol{R}$ value increases from $0.000039 B^{2}$ to $0.6 B^{2}$, after that it begins to increase to $95 \%$ as the $\boldsymbol{R}$ increases to $3 B^{2}$, then it almost keep around this percentage with larger values for $\boldsymbol{R}$. A possible reason is that the Kalman gain step becomes small as the $\boldsymbol{R}$ value increases.
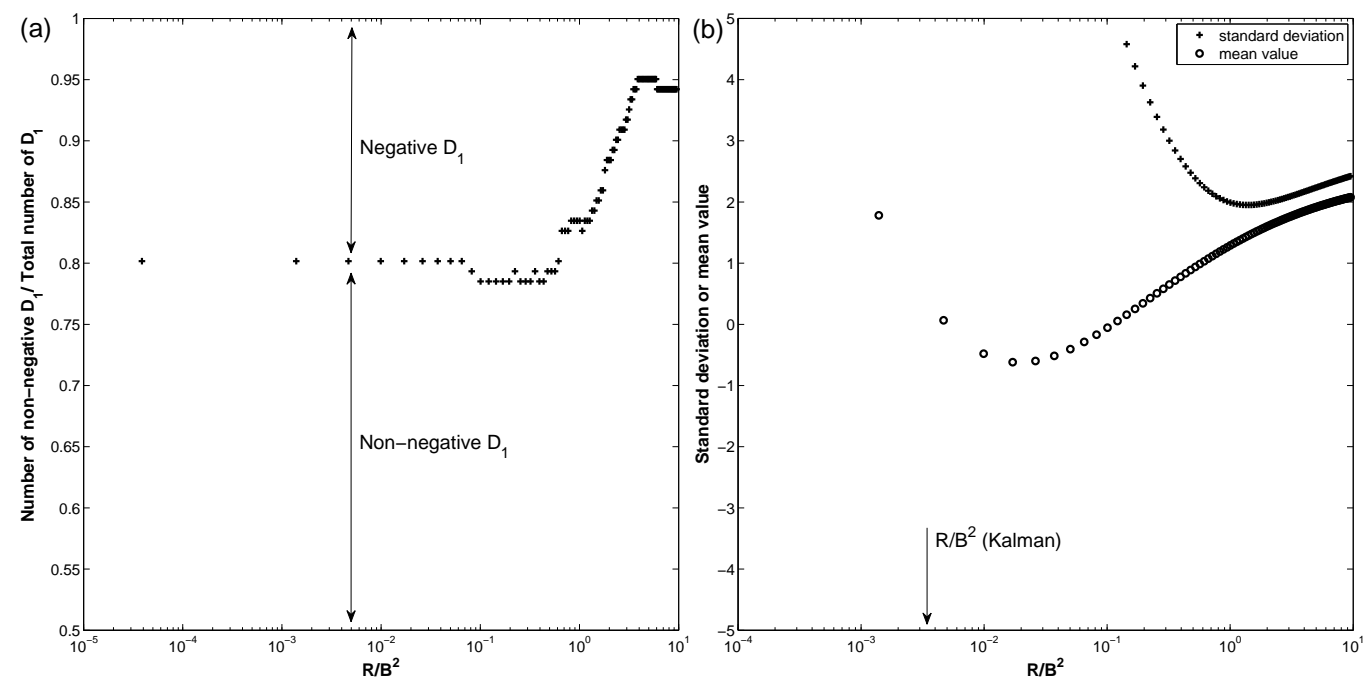

Fig. 3. Effect of $\boldsymbol{R}$ on $D_{1} / D$. (a) convergent of $D_{1} / D$ over total number of $D_{1} / D$. (b) standard deviation and mean value, the standard deviation values over 5 are excluded from the graph. $\boldsymbol{Q}=0$.

The effect of $\boldsymbol{R}$ on standard deviations and mean values are shown in Fig. 3(b). The figure shows that the standard deviation decreases as the $\boldsymbol{R}$ increases untill $\boldsymbol{R}=1.5 B^{2}$, then it increases slowly, also the mean value decreases as the $\boldsymbol{R}$ increases to $0.02 B^{2}$, and then increases again. The $\boldsymbol{R} / B^{2}$ (Kalman) in the graph denoted suggested $\boldsymbol{R}$ for ordinary Kalman filter based on the average value of $z$ and the $10 \%$ measurements noise, i.e $\boldsymbol{R}=\left(0.1 z_{a v}\right)^{2}$, which is not the optimal case. One reason is that standard deviations and mean values are computed after one iteration, also the negative values of $D$ are included. Slightly different results is obtained after more iterations, reason should be the non-linear model.

Fig. 4(a) shows the effect of different values of $Q$ using $0.01 \leq p \leq 1$ and $\boldsymbol{R}$ as given in Eq. (16) on the convergent numbers of $D_{5} / D$ over total number of $D_{5} / D$. It is clearly seen that the number of convergent $D_{5} / D$ decreases as the $p$ increases. Using large $\boldsymbol{Q}$ with small $\boldsymbol{R}$ affects the Kalman gain stability, this could be a possible reason. 



Fig. 4. The effect of $p$ on $D_{5} / D$. (a) on convergent of $D_{5} / D$ over total number of $D_{5} / D$ (b) standard deviation and mean value of $D_{5} / D . R$ is chosen as in Eq. (16).

Fig. 4(b) shows the effect of $p$ on the standard deviations and mean values for the obtained $D_{5} / D$. The figure shows that standard deviation increases as $p$ increases, and mean values play around 1.7 as the $p$ increases. One reason could be that using large values of $\boldsymbol{Q}$ with small values for $\boldsymbol{R}$ increases the Kalman gain step. The $\boldsymbol{Q}$ value at $p=0.71$, random walk (cf. [2]), and at $p=1$, the suggested variance as in Eq. (13), does not give significant effect on both standard deviation and mean value. A non-linear model might be a reason, so it is expected to have effect with more iterations. The $p$ value is chosen for 0.01 that give smallest standard deviation and largest percentage of number of convergent $D$ over total number of $D$ to be one method (see Table 1).

\subsection{Large $\boldsymbol{R}$ and $\boldsymbol{Q}$}

By studying Eqs (7) and (9), and Fig. 3 it is believed that choosing $\boldsymbol{R}$ large, decreases the risk of ending up with divergent results for $D_{k}$. Fig. 5(a) shows that increasing $p$ for large values using $\boldsymbol{R}$ large increases the percentage of the number of convergent $D_{5}$ by $3 \%$, this is an indication that using $p>0$ with $\boldsymbol{R}$ large covers a wide range of initial parameters that converges. 

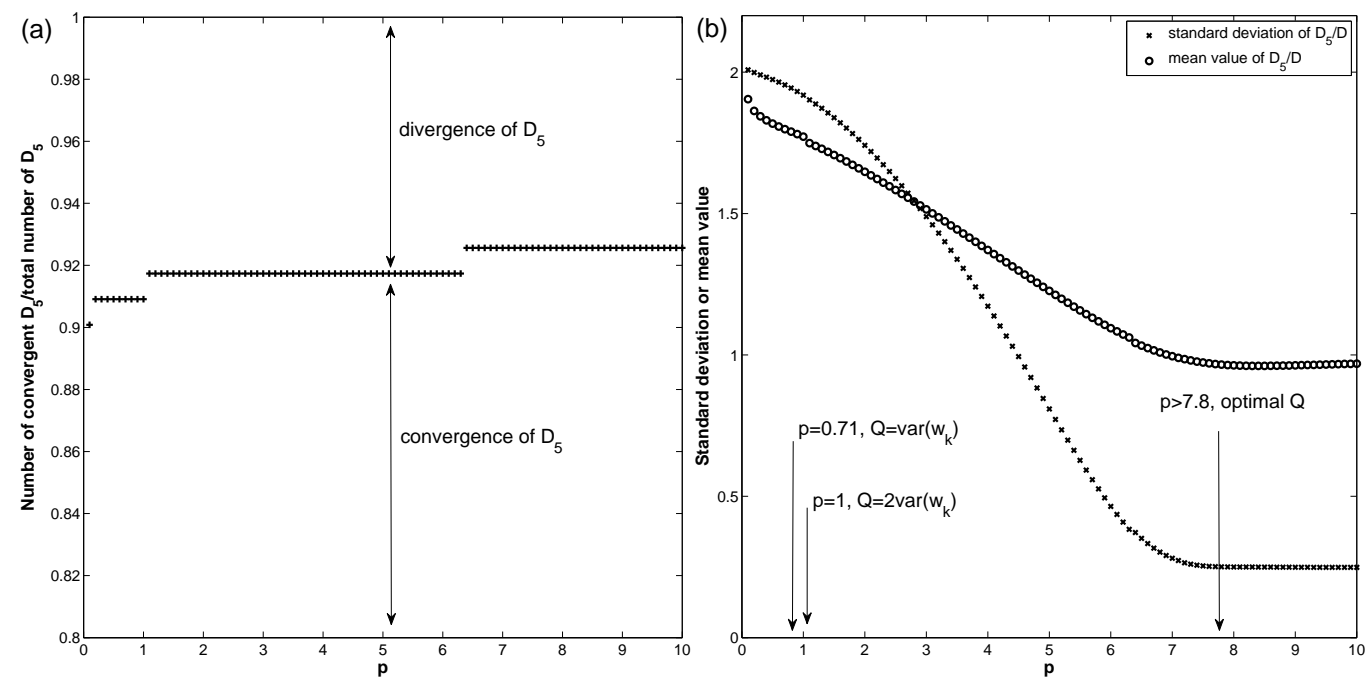

Fig. 5. The effect of $p$ on $D_{5} / D$ using $R$ large. (a) convergent of $D_{5} / D$ over total number of $D_{5} / D$ (b) standard deviation and mean value of $D_{5} / D$. The large $\boldsymbol{R}$ is chosen as in Eq. (26).

The effect of $\boldsymbol{Q}$ on the standard deviations and mean values of the obtained $D_{5} / D$ are shown in Fig. 5(b). It can be seen that the standard deviation and mean value decrease as the $p$ increases, untill optimal is obtained with $p>7.8$. Also, increasing $p$ speeds the rate of convergence. Again, this could be the effect of non-linearity of the model.

It can be concluded that using $\boldsymbol{R}$ and $\boldsymbol{Q}$ large increase the possibility of convergence for a wide range of $D_{0}$ and $B_{0}$ and speeds the convergence of parameters. To choose $\boldsymbol{R}$ for large value, a vector $v_{s}$ contains the largest variance value between measurement, $z$, and predicted, $h\left(x_{0}\right)$, for each initial parameters is chosen as

$v_{s}=\left[v^{(1)} v^{(2)} \ldots v^{(1681)}\right]^{T}$.

where $s$ is the number of initial parameters, i.e $s=1,2, \ldots ., 1681$, and $v^{(i)}$ values are given by Eq. (15), then the $\boldsymbol{R}$ value is chosen as the maximum value in the whole initial parameters combinations as

$$
\boldsymbol{R}=\max \left(v_{s}\right) I,
$$

To choose $\boldsymbol{Q}$ large, the $\boldsymbol{Q}$ is chosen to be equal to $\boldsymbol{P}_{0}^{-}$as in Eq. $(23)$, since the $Q$ represents the variance between the seeking parameters and instant parameters. 
Fig. 6(a) shows that the divergent result after 1 iteration using large $\boldsymbol{R}$ and $\boldsymbol{Q}=$ 0 decreased to $7.8 \%$ compared with the divergence result of $21.5 \%$ for different $\boldsymbol{R}$ values as shown in Fig. 2(a). It also decreased to $7.9 \%$ after 20 iterations using large $\boldsymbol{R}$ and $\boldsymbol{Q}$ as shown in Fig. 6(b) compared to the divergence result of $22.4 \%$ for different $\boldsymbol{R}$ and $\boldsymbol{Q}$ values as shown in Fig. 2(b).

It can be discussed that large values of $\boldsymbol{R}$ and $\boldsymbol{Q}$ decreases the percentage of divergent result of $D_{k}$, and also speeds the rate of convergence as shown in Fig. 2 and Fig. 6.
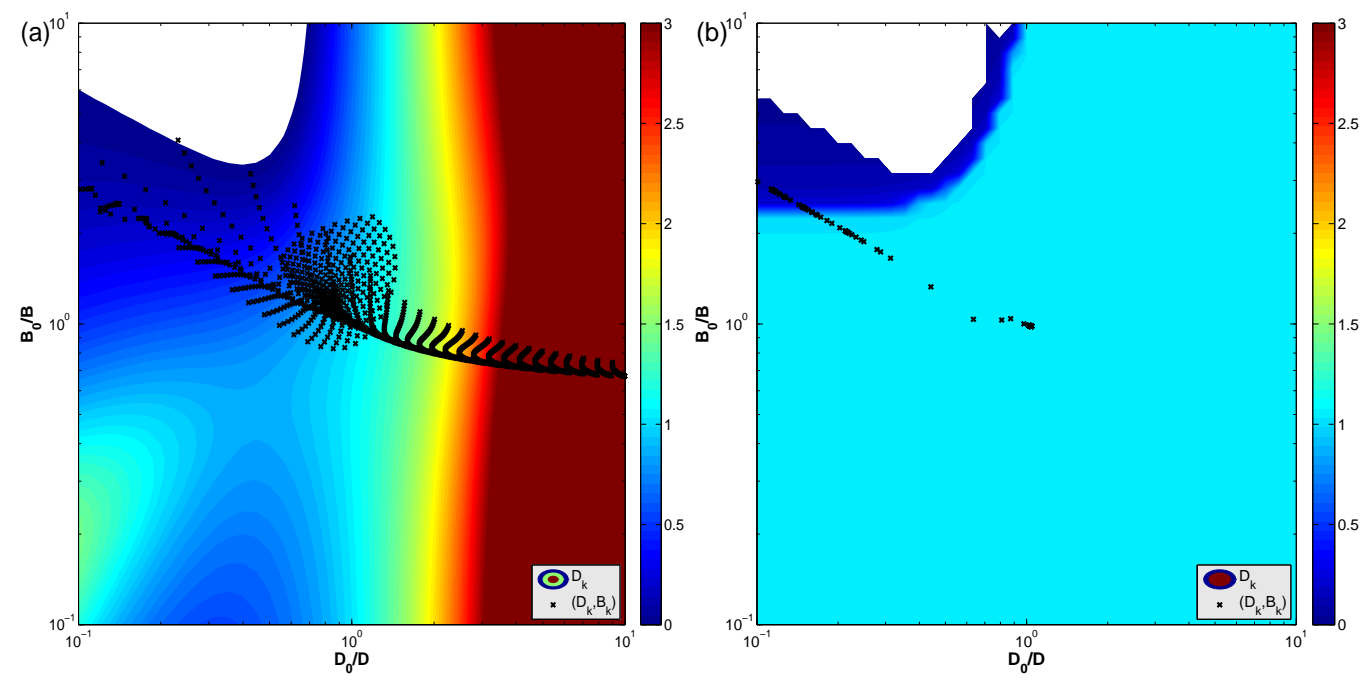

Fig. 6. Color plot for $D_{k}$ merged with convergence plot between $\left(D_{k}, B_{k}\right)$ for initial starting points $D_{0}$ and $B_{0}$ after (a) 1 iteration, for large $\boldsymbol{R}$ and $\boldsymbol{Q}=0$, and (b) 20 iterations for large $\boldsymbol{R}$ and large $\boldsymbol{Q} \cdot \boldsymbol{Q}$ is selected to be large and equal to $\boldsymbol{P}_{0}^{-}$ as in Eq. (23), while $\boldsymbol{R}$ is chosen as in Eq. (26). Noises are $\pm 5 \%$ for the parameters and $\pm 10 \%$ for the measurements.

\subsection{Different methods}

The method with the selections of $\boldsymbol{Q}$ and $\boldsymbol{R}$, is compared with with the second, the third, and the fourth method that are explained in subsection subsection 3.1. and summarized in 1 . The $\boldsymbol{R}$ in the second and third methods is chosen as the maximum difference between generated data, $z$, and generated data with the noises, $h(x)$. The $\boldsymbol{P}_{0}^{-}$for the second and third method is chosen as in Eq. (23), the same as in the suggested method. Fig. 7(a) shows that $92.1 \%$ of the initial selected $D_{0} / D$ and $B_{0} / B$ give convergent results for diffusion constants. Around $98.3 \%$ of those 
values are converged to 1.042 after 50 iterations, while the $1.7 \%$ left (blue area in the color plot) almost converged to the same point after 180 iterations. The Kalman filter with $p=0.01$ gives almost the same convergent area of $D_{50} / D$ as the Kalman filter with $p=0$ around $80 \%$, but it speeds the rate of convergence as shown in Fig. 7(c), while Kalman filter with $p=0$ needs a large number of iteration for the parameters to converge as shown in Fig. 7(b). The non-linear least square speeds the rate of convergence but with $40.93 \%$ of the initial selected parameters that give convergent results for diffusion constants as shown in Fig. 7(d). 

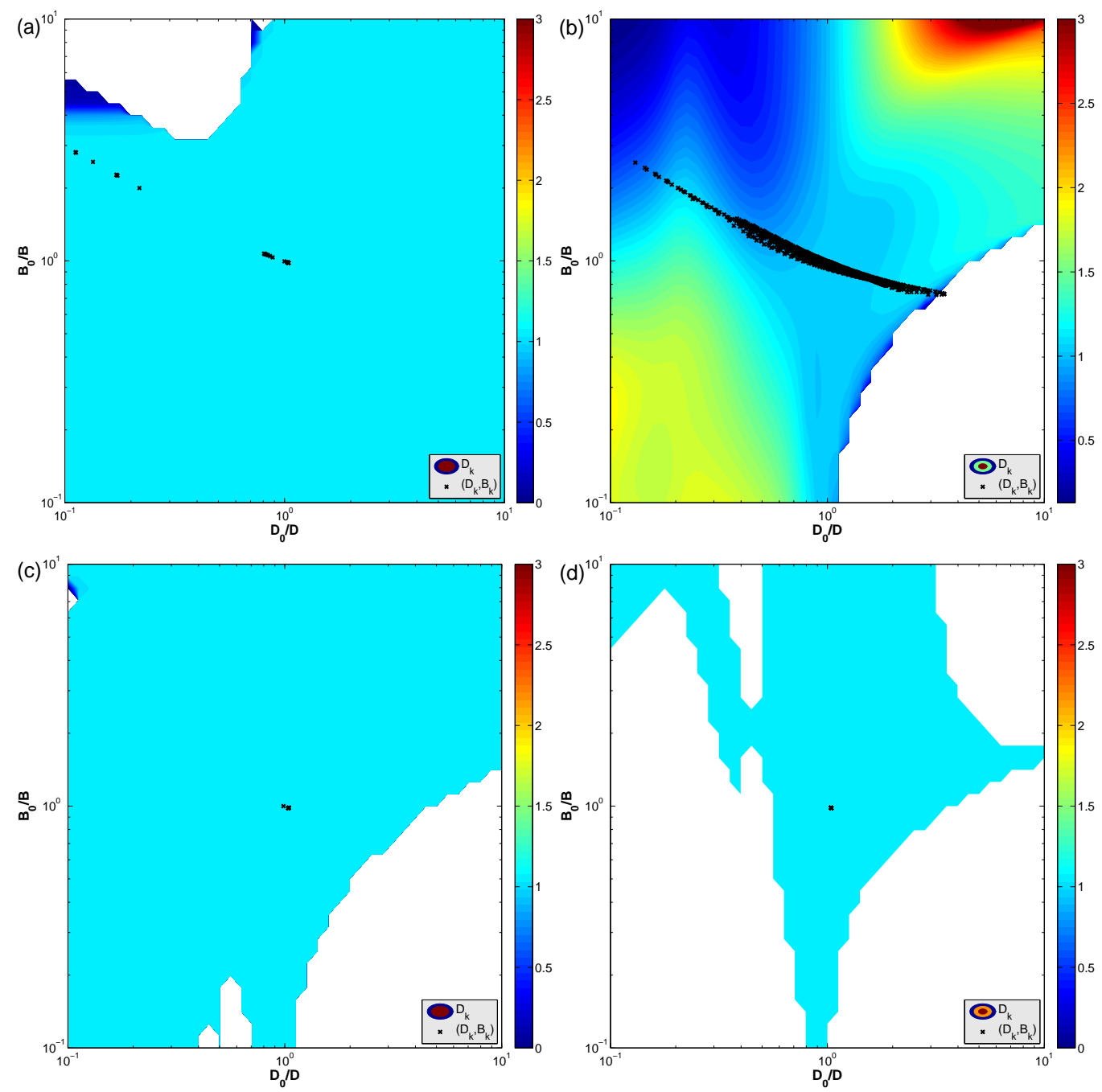

Fig. 7. Color plot for $D_{50} / D$ merged with convergence plot between $\left(D_{50} / D, B_{50} / B\right.$ ) for initial starting points $D_{0} / D$ and $B_{0} / B$ after 50 iterations for: (a) suggested Kalman filter with large $\boldsymbol{R}$ and large $\boldsymbol{Q}$, (b) Kalman filter with $p=0$, (c) Kalman filter with $p=0.01$, and (d) non-linear least square method. For generated data with noises of $\pm 5 \%$ for parameters and $\pm 10 \%$ for measurements.

The suggested Kalman filter covers a wide range of the initial selected values for $D_{0}$ and $B_{0}$ that give convergent results for diffusion $D_{50}$ compared with Kalman filter with $p=0$, Kalman filter with $p=0.01$, and non-linear least square. This 
makes it an appropriate method to determine diffusion coefficient if the a priori information is rare or there is a large variation in the parameters, such as bone as an inhomogeneous material. Also, it speeds the rate of convergence. The Kalman filter with $p=0.01$ might be a good choice if the a priori information in the adjacent area of the seeking parameters are enough. Also, the non-linear least square might be one choice but for small range of initial selected values for $D_{0}$ and $B_{0}$.

The effect of different ranges for $D_{0}$ and $B_{0}$ against the standard deviations and mean values for the four methods after 5 iterations are shown in Fig. 8. The ranges are selected to start from a small range and end with a large range as

$$
\begin{aligned}
\frac{1}{i D}<\text { selected initial ranges }<i D & \\
\frac{1}{i B}<\text { selected initial ranges }<i B &
\end{aligned}
$$

where $i=1.2,1.6,2, \ldots . ., 10$. The figure shows that the standard deviations and mean values increase as $i$ increases. A reason for that could be that the number of initial parameters increases as the ranges increase. It can be noticed that Kalman filter with $p=0.01$ gives the smallest standard deviations while the suggested method gives standard deviations closest to Kalman filter with $p=0$. On the other hand, the mean values obtained by suggested method is found to be the smallest among the other three methods. This is an indication that the suggested method and Kalman filter with $p=0.01$ might converge around the same speed. On the contrary, the non-linear least square gives very large values for standard deviations for $i>1.6$, these values are above 2 and excluded from the graph as shown in Fig. 8(a), and gives large mean values for $D_{5} / D$ as shown in Fig. 8(b). A possible reason for that is the divergent result that obtained after 5 iterations, it has some large negative values for $D_{5} / D$. 

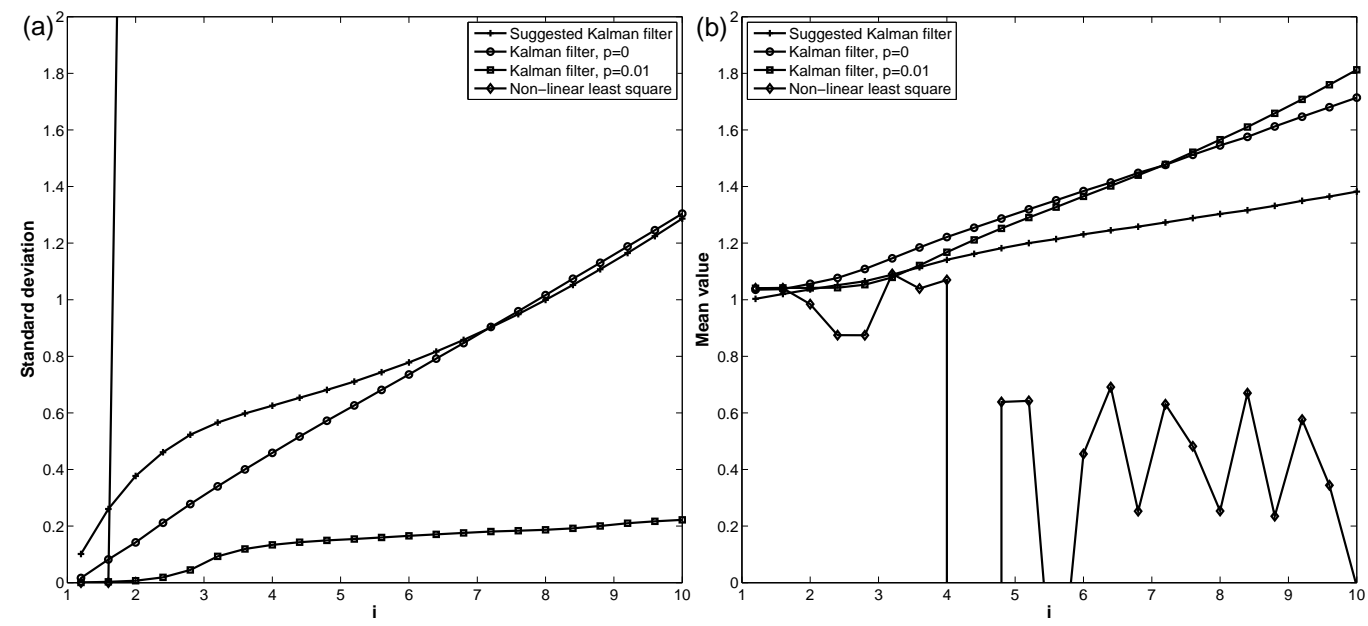

Fig. 8. Standard deviations and mean values for obtained $D_{5} / D$ against ranges of selected initial parameters for the four methods after 5 iterations, (a) standard deviation and (b) mean value. For generated data with noises of $\pm 5 \%$ for parameters and $\pm 10 \%$ for measurements.

Fig. 9 shows the standard deviations and mean values of the obtained $D_{k}$, $k=1,2, \ldots ., 100$ against number of iterations for large initial range, $i=10$, for the four methods. The standard deviation values for $D_{k}$ for the suggested Kalman filter decrease slowly up to 70 iterations, and the mean value increases as number of iterations increases to 70 iterations, and almost stable after that. The reason for that is some of the obtained values of $D_{k}$ were stuck to small values up to the 70 iterations, moving with small steps of $\boldsymbol{K}_{k}$ until they reached to good predicted values to move with larger steps. 

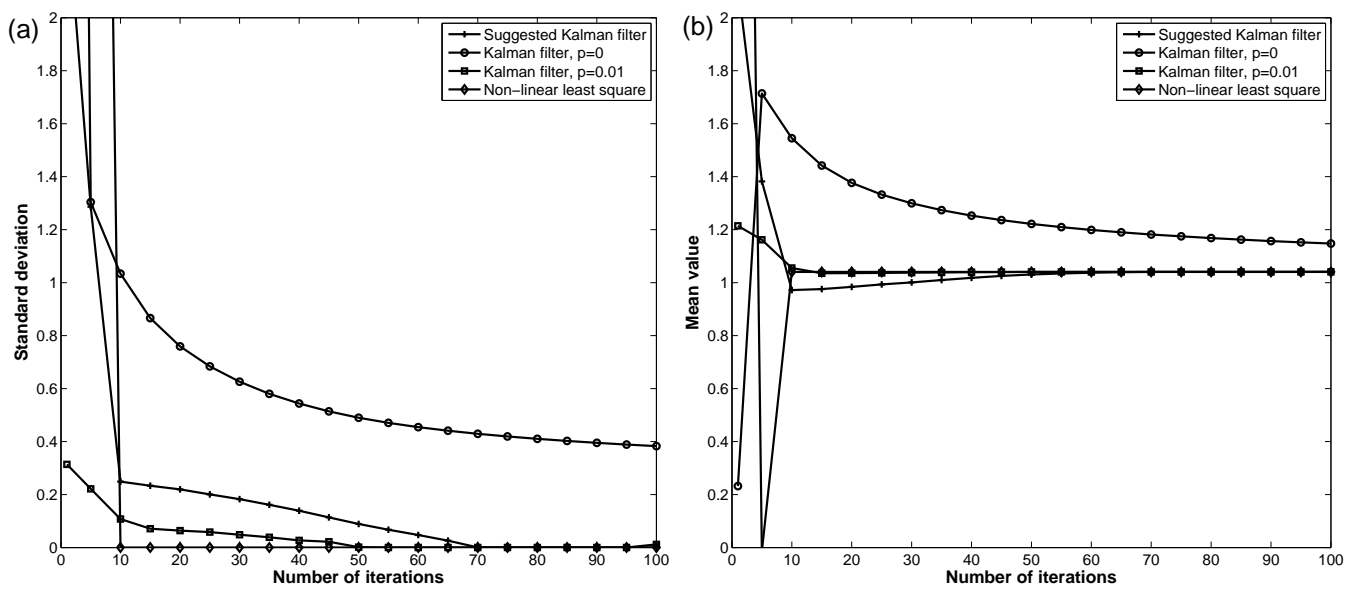

Fig. 9. Standard deviations and mean values for obtained $D_{k}$ against number of iterations for the four methods after 5 iterations, (a) standard deviation and (b) mean value. For generated data with noises of $\pm 5 \%$ for parameters and $\pm 10 \%$ for measurements. ( points over 2 and less than 0 are excluded from the graph).

The standard deviation and mean value using the Kalman filter with $p=0$ decrease slowly as the number of iterations increases. This is expected since $\boldsymbol{R}$ in the second method was chosen to a small value and $\boldsymbol{Q}$ vanishes, which makes the Kalman gain is small. On the other hand, the standard deviation and mean values of $D_{k}$ for Kalman filter with $p=0.01$ and for non-linear least square converge quicker than the others. This is an indication that methods 3 and 4 can be used effectivly if the apriori information fortunately adjacent to the seeking parameters.

The determination of diffusion constants for generated data with $\pm 5 \%$ parameters noise and $\pm 50 \%$, and $\pm 100 \%$ measurements noise almost follow the same trend as in $\pm 5 \%$ parameters noise and $\pm 10 \%$ measurements noise. Table 2 shows the standard deviation, mean, and the percentage of numbers of convergent $D_{50} / D$ over total number of $D_{50} / D(\mathrm{D} . \mathrm{N} \%)$ for the selected range with $i=10$, for the four methods. 
Table 2: Standard deviations, mean values and D.N\% for the obtained $D_{50} / D$ for the four methods with noises of $\pm 5 \%$ for parameters and $\pm 10 \%, \pm 50 \%$, and $\pm 100 \%$ for measurements.

\begin{tabular}{ccccc}
\hline Random noises & Method & Standard deviation & Mean & D.N\% \\
\hline $\pm 10 \%$ & Suggested Kalman filter & 0.09 & 1.03 & 92.09 \\
& Kalman filter, $p=0$ & 0.49 & 1.22 & 80.90 \\
& Kalman filter, $p=0.01$ & 0.03 & 1.04 & 80.19 \\
& Non-linear least square & $8.71 \times 10^{-09}$ & 1.04 & 40.93 \\
$\pm 50 \%$ & Suggested Kalman filter & 0.06 & 0.93 & 91.96 \\
& Kalman filter, $p=0$ & 0.54 & 1.06 & 80.37 \\
& Kalman filter, $p=0.01$ & 0.003 & 0.94 & 80.67 \\
& Non-linear least square & $6.19 \times 10^{-16}$ & 0.94 & 45.63 \\
& Suggested Kalman filter & 0.03 & 1.69 & 92.03 \\
& Kalman filter, $p=0$ & 0.66 & 1.44 & 85.24 \\
& Kalman filter, $p=0.01$ & 0.22 & 1.66 & 85.66 \\
& Non-linear least square & 0.064 & 1.69 & 42.47 \\
\hline
\end{tabular}

The table shows that the suggested Kalman filter gives a compromise results compared with the other methods. The most important difference is the percentage of obtained $D_{50} / D$ that give convergent results (around 92\%), that is found to be large compared with the others, which means high possibilities to determine the diffusion coefficients from the selected initial range even it was a large range, and for measurements noise up to $\pm 100 \%$. The table also shows that the suggested Kalman filter speeds the rate of convergence as well as Kalman filter with $p=0.01$ and non-linear least square but with higher D.N\%. By comparing standard deviations and mean values as in the table, the standard deviations for Kalman filter with $p=0$ give the largest values among the other methods, and the mean values are pretty a way from the convergent parameters. A reason for that is Kalman filter with $p=0$ converges to many different points with small Kalamn gain step, which means more iterations are needed for convergence. In sum, the suggested method can be applied effectively for both rare and sufficient information about the seeking parameters, and can be applied for a wide range of initial parameters.

The behavior of mean square error for a selected real bovine bone sample is found almost the same as the generated one in Fig. 1. The initial predicted parameters $D_{0}$ and $B_{0}$ are chosen to be $41 \times 41$ combinations, with $0.001<D_{0}<0.1$ 
$\mathrm{mm}^{2} / \mathrm{min}$ and $6<B_{0}<600 \mu \mathrm{S} / \mathrm{mm}$, which constructs a large combinations between the initial parameters. The initial predicted parameters error $\boldsymbol{P}_{0}^{-}$, covariance error for the parameters $\boldsymbol{Q}$, and covariance error for the measurements $\boldsymbol{R}$ are chosen based on both suggested method and these combinations. Fig. 10 shows the convergence plot for the two parameters $D$ and $B$ that obtained using the suggested Kalman filter for the real sample after 1, 10, 50, and 250 iterations.



Fig. 10. Convergence between $D_{k}$ and $B_{k}$ obtained using suggested method for a real sample for $1,10,50$, and 250 iterations

The initial predicted parameters that give convergent results for $D_{k}$ are found to be $92.2 \%$ from the combination, that converged around a specific line after some iterations as in 10 and 50 iterations (see Fig. 10). Around 92.5\% of them converged to the seeking unknown parameters $D=0.0144 \mathrm{~mm}^{2} / \mathrm{min}$ and $B=$ $73.602 \mu \mathrm{S} / \mathrm{mm}$ after 50 iterations, while the $7.5 \%$ left converged to the same place almost after 250 iterations. Small Kalman gain steps at the $7.5 \%$ might be a possible reason for the slowly convergence. The convergence line is expected since the mean square error has smaller values along this line as shown in Fig. 1.

The conductivity versus time for analytical model Eq. (20) using $D$ and $B$ obtained by the suggested Kalman filter and experimental data for the real sample is shown in Fig. 11. The figure shows that the analytical function fits very well with the experimental data accompanied with mean square error of $0.85(\mu \mathrm{S} / \mathrm{mm})^{2}$. 


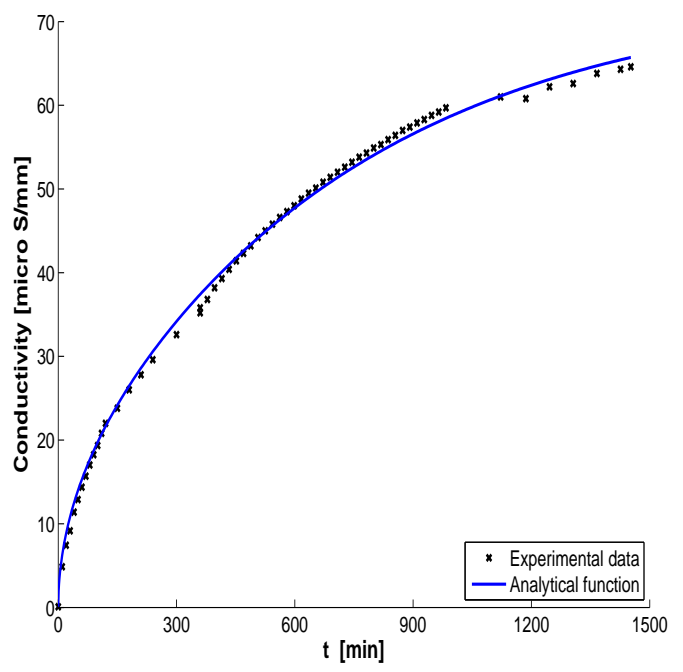

Fig. 11. Conductivity versus time for analytical and experimental for the real sample

\section{Conclusions}

Four methods to choose appropriate initial parameters, covariance errors for parameters, and covariance errors for measurements, required for Kalman filter for determination of material parameters, is investigated in this work. The methods are applied to generated data with $\pm 5 \%$ parameters noise and $\pm 10 \%, \pm 50 \%$, and $\pm 100 \%$ measurements noise for known parameters. The suggested method covers a wider range of the initial suggested values for the parameters than the standard Kalman filters, and non-linear least square, which enhances the possibilities of convergence around the seeking parameters. The suggested method speeds the rate of convergence compared with the other methods. Very good results are obtained for diffusion coefficient in bovine bone as a case study.

\section{Appendix A. Non-linear least squares}

The sum of the squared residuals is written

$\Phi=(z-h)^{\mathrm{T}}(z-h)$ 
By putting the derivative of $\Phi$ with respect to $x$ to zero, a set of $n$ equations is obtained,

$$
\frac{\partial \Phi}{\partial x}=0 \Rightarrow \mathbf{H}(x)^{\mathrm{T}}\{z-h(x)\}=0 .
$$

Insertion of Eq. (3) into Eq. (A-2) gives the following,

$$
\mathbf{H}^{\mathrm{T}}\left\{z-h\left(\hat{x}_{k}\right)\right\} \approx \mathbf{H}^{\mathrm{T}} \mathbf{H}\left(x-\hat{x}_{k}\right) .
$$

Provided that the $n \times n$ matrix $\mathbf{H}^{\mathrm{T}} \mathbf{H}$ is non-singular, one obtains the following approximation,

$x \approx \hat{x}_{k}+\left(\mathbf{H}^{\mathrm{T}} \mathbf{H}\right)^{-1} \mathbf{H}^{\mathrm{T}}\left\{z-h\left(\hat{x}_{k}\right)\right\}$.

For linear systems the Jacobian $\mathbf{H}$ is independent of $x$, which makes the solution in Eq. (A-4) exact.

\section{Appendix B. Kalman filter derivation}

The variance of the errors of the parameters before, $\boldsymbol{P}_{k}^{-}$, and after, $\boldsymbol{P}_{k}$, the iterative update are

$\boldsymbol{P}_{k}^{-}=\operatorname{Var}\left(x_{k}-\hat{x}_{k-1}\right)$, and $\boldsymbol{P}_{k}=\operatorname{Var}\left(x_{k}-\hat{x}_{k}\right)$.

The function $\operatorname{Var}(a)$ is an $l \times l$ matrix with the elements $\alpha_{i j}$ given by the vector $a=\left(a_{1}, \ldots a_{l}\right)^{\mathrm{T}}$ as follows (cf. [20])

$\alpha_{i j}=\mathrm{E}\left(x_{i} x_{j}\right)-\mathrm{E}\left(x_{i}\right) \mathrm{E}\left(x_{j}\right)$,

where $E(\vartheta)$ is the statistical mean value of the stochastic variable $\vartheta$. Hence, the matrices $\boldsymbol{P}_{k}$ and $\boldsymbol{P}_{k-1}^{-}$are symmetric with the dimension $n \times n$.

Using Eqs. (B-1) and (2), a relation between $\boldsymbol{P}_{k}^{-}$and $\boldsymbol{P}_{k}$ is obtained as,

$$
\begin{aligned}
\boldsymbol{P}_{k+1}^{-} & =\operatorname{Var}\left(x_{k+1}-\hat{x}_{k}\right) \\
& =\operatorname{Var}\left(x+w_{k+1}-\hat{x}_{k}\right) \\
& =\operatorname{Var}\left(x_{k}-w_{k}+w_{k+1}-\hat{x}_{k}\right) \\
& =\operatorname{Var}\left(x_{k}-\hat{x}_{k}\right)+\operatorname{Var}\left(-w_{k}+w_{k+1}\right) \\
& =\operatorname{Var}\left(x_{k}-\hat{x}_{k}\right)+2 \operatorname{Var}\left(w_{k}\right) \\
& =\boldsymbol{P}_{k}+\boldsymbol{Q}_{k}
\end{aligned}
$$


where $\boldsymbol{Q}_{k}=2 \operatorname{Var}\left(w_{k}\right) \boldsymbol{Q}_{k}$ is an $n \times n$ matrix. Note that the covariance of the supposedly uncorrelated quantities $\left(x-\hat{x}_{k}\right)$ and $w_{k}$ respectively vanishes.

On the other hand, substituting $\hat{x}_{k}$ in Eq. (B-1) by using Eq. (6), $\boldsymbol{P}_{k}$ can be expressed as

$\boldsymbol{P}_{k}=\operatorname{Var}\left[x_{k}-\left(\hat{x}_{k-1}+\mathbf{K}_{k}\left\{z_{k}-h\left(\hat{x}_{k-1}\right)\right\}\right)\right]$.

Replacing $z_{k}$ according to Eq. (1) leads to

$$
\begin{aligned}
\boldsymbol{P}_{k}= & \operatorname{Var}\left(x_{k}-\hat{x}_{k-1}-\mathbf{K}_{k}\left\{h\left(x_{k}\right)+v_{k}-h\left(\hat{x}_{k-1}\right)\right\}\right) \\
= & \operatorname{Var}\left(x_{k}-\hat{x}_{k-1}\right)-\mathbf{K}_{k} \operatorname{Cov}\left(h\left(x_{k}\right)-h\left(\hat{x}_{k-1}\right), x_{k}-\hat{x}_{k-1}\right) \\
& -\operatorname{Cov}\left(x_{k}-\hat{x}_{k-1}, h\left(x_{k}\right)-h\left(\hat{x}_{k-1}\right)\right) \mathbf{K}_{k}^{\mathrm{T}} \\
& +\mathbf{K}_{k} \operatorname{Var}\left(h\left(x_{k}\right)-h\left(\hat{x}_{k-1}\right)\right) \mathbf{K}_{k}^{\mathrm{T}} \\
& +\mathbf{K}_{k} \operatorname{Var}\left(v_{k}\right) \mathbf{K}_{k}^{\mathrm{T}},
\end{aligned}
$$

where the function $\operatorname{Cov}(a, b)$ gives the covariance of the stochastic vectors $a$ and $b$. The function $\operatorname{Cov}(a, b)$ is an $m \times m$ matrix with the elements $\alpha_{i j}$ given by the stochastic variables $a=\left(a_{1}, \ldots a_{m}\right)^{\mathrm{T}}$ and $b=\left(b_{1}, \ldots b_{m}\right)^{\mathrm{T}}$ as follows (cf. [20]),

$\alpha_{i j}=\mathrm{E}\left(a_{i} b_{j}\right)-\mathrm{E}\left(a_{i}\right) \mathrm{E}\left(b_{j}\right)$.

It is used that the covariance between the elements of $h\left(x_{i}\right)$ and $v_{k}$ for any $i$ and $k$ vanishes. The Taylor series in Eq. (3) giving $h\left(x_{k}\right)-h\left(\hat{x}_{k-1}\right)=\mathbf{H}_{k}\left(x_{k}-\hat{x}_{k-1}\right)$ results in

$$
\begin{aligned}
\boldsymbol{P}_{k} & =\boldsymbol{P}_{k}^{-}-\mathbf{K}_{k} \mathbf{H}_{k} \boldsymbol{P}_{k}^{-}-\boldsymbol{P}_{k}^{-} \mathbf{H}_{k}^{\mathrm{T}} \mathbf{K}_{k}^{\mathrm{T}}+\mathbf{K}_{k} \mathbf{H}_{k} \boldsymbol{P}_{k}^{-} \mathbf{H}_{k}^{\mathrm{T}} \mathbf{K}_{k}^{\mathrm{T}}+\mathbf{K}_{k} \boldsymbol{R}_{k} \mathbf{K}_{k}^{\mathrm{T}} \\
& =\left(\boldsymbol{I}-\mathbf{K}_{k} \mathbf{H}_{k}\right) \boldsymbol{P}_{k}^{-}\left(\boldsymbol{I}-\mathbf{K}_{k} \mathbf{H}_{k}\right)^{\mathrm{T}}+\mathbf{K}_{k} \boldsymbol{R}_{k} \mathbf{K}_{k}^{\mathrm{T}},
\end{aligned}
$$

where $R_{k}$ is the covariance error of the measurements and an $N \times N$ matrix defined as follows

$\boldsymbol{R}_{k}=\operatorname{Var}\left(v_{k}\right)$.

Since the $x_{k}$ and $v_{k}$ are mutually uncorrelated, $\boldsymbol{P}_{k}$ becomes a diagonal matrix that contains errors between the parameters before and after an iteration, the $K_{k}$ that minimizes the error can be obtained by taking the derivative of the trace $\operatorname{Tr}\left(\boldsymbol{P}_{k}\right)$ with respect to $\mathbf{K}_{k}$ and putting it equal to zero. Taking the trace of the first equality in Eq. (B-6) provides 
$\operatorname{Tr}\left(\boldsymbol{P}_{k}\right)=\operatorname{Tr}\left(\boldsymbol{P}_{k}^{-}\right)-\operatorname{Tr}\left(\mathbf{K}_{k} \mathbf{H}_{k} \boldsymbol{P}_{k}^{-}\right)-\operatorname{Tr}\left(\boldsymbol{P}_{k}^{-} \mathbf{H}_{k}^{\mathrm{T}} \mathbf{K}_{k}^{\mathrm{T}}\right)+\operatorname{Tr}\left(\mathbf{K}_{k} \mathbf{H}_{k} \boldsymbol{P}_{k}^{-} \mathbf{H}_{k}^{\mathrm{T}} \mathbf{K}_{k}^{\mathrm{T}}\right)+\operatorname{Tr}\left(\mathbf{K}_{k} \boldsymbol{R}_{k} \mathbf{K}_{k}^{\mathrm{T}}\right)$

Using following identities for the matrix trace (cf. [21] )

$\operatorname{Tr}\left(\boldsymbol{P}_{k}^{-} \mathbf{H}_{k}^{\mathrm{T}} \mathbf{K}_{k}^{\mathrm{T}}\right)=\operatorname{Tr}\left(\mathbf{K}_{k} \mathbf{H}_{k} \boldsymbol{P}_{k}^{-}\right), \frac{\partial \operatorname{Tr}(\boldsymbol{A} \boldsymbol{C})}{\partial \boldsymbol{A}}=\boldsymbol{C}^{T}$ and $\frac{\partial \operatorname{Tr}\left(\boldsymbol{A} \boldsymbol{F} \boldsymbol{A}^{T}\right)}{\partial \boldsymbol{A}}=2 \boldsymbol{A} \boldsymbol{F}$.

The last equality requires that the matrix $\boldsymbol{F}$ is symmetric. The derivative of Eq. (B-8) with respect to $\mathbf{K}_{k}$ can be written

$\frac{\partial \operatorname{Tr}\left(\boldsymbol{P}_{k}\right)}{\partial \mathbf{K}_{k}}=-2 \boldsymbol{P}_{k}^{-} \mathbf{H}_{k}^{\mathrm{T}}+2 \mathbf{K}_{k} \mathbf{H}_{k} \boldsymbol{P}_{k}^{-} \mathbf{H}_{k}^{\mathrm{T}}+2 \mathbf{K}_{k} \boldsymbol{R}_{k}$

The optimal $\mathbf{K}_{k}$ can be obtained by putting the right term in Eq. [B-10] to zero as next

$\mathbf{K}_{k}=\boldsymbol{P}_{k}^{-} \mathbf{H}_{k}^{\mathrm{T}}\left(\mathbf{H}_{k} \boldsymbol{P}_{k}^{-} \mathbf{H}_{k}^{\mathrm{T}}+\boldsymbol{R}_{k}\right)^{-1}$.

The $\boldsymbol{P}_{k}$ associated to the optimal $\mathbf{K}_{k}$ can be written as in Eq. [B-12] by substituting Eq. [B-11] into Eq. (B-6) as next

$\boldsymbol{P}_{k}=\left(\boldsymbol{I}-\mathbf{K}_{k} \mathbf{H}_{k}\right) \boldsymbol{P}_{k}^{-}+\left\{-\boldsymbol{P}_{k}^{-} \mathbf{H}_{k}^{\mathrm{T}}+\mathbf{K}_{k}\left(\mathbf{H}_{k} \boldsymbol{P}_{k}^{-} \mathbf{H}_{k}^{\mathrm{T}}+\boldsymbol{R}_{k}\right)\right\} \mathbf{K}_{k}^{\mathrm{T}}$

Using Eq. (B-11) readily gives

$\boldsymbol{P}_{k}=\left(\boldsymbol{I}-\mathbf{K}_{k} \mathbf{H}_{k}\right) \boldsymbol{P}_{k}^{-}$,

and consequently

$\boldsymbol{P}_{k+1}^{-}=\left(\boldsymbol{I}-\mathbf{K}_{k} \mathbf{H}_{k}\right) \boldsymbol{P}_{k}^{-}+\boldsymbol{Q}_{k}$,

\section{References}

\section{References}

[1] R. E. Kalman, A new approach to linear filtering and prediction problems, Journal of Fluids Engineering 82 (1) (1960) 35-45. doi : 10.1115/1.3662552.

[2] R. G. Brown, Introduction to random signal analysis and Kalman filtering, Vol. 8, Wiley New York, 1983.

[3] G. M. Siouris, G. Chen, J. Wang, Tracking an incoming ballistic missile using an extended interval kalman filter, Aerospace and Electronic Systems, IEEE Transactions on 33 (1) (1997) 232-240. doi:10.1109/7.570753. 
[4] S.-K. Weng, C.-M. Kuo, S.-K. Tu, Video object tracking using adaptive kalman filter, Journal of Visual Communication and Image Representation 17 (6) (2006) 1190-1208. doi:10. $1016 / j$.jvcir.2006.03.004

[5] S. Antonov, A. Fehn, A. Kugi, Unscented kalman filter for vehicle state estimation, Vehicle System Dynamics 49 (9) (2011) 1497-1520. doi:10. 1080/00423114.2010.527994.

[6] K. Ahn, D. Truong, Online tuning fuzzy pid controller using robust extended kalman filter, Journal of Process Control 19 (6) (2009) 1011-1023. doi:10.1016/j . jprocont.2009. Q1.005.

[7] Y. Shi, H. Fang, M. Yan, Kalman filter-based adaptive control for networked systems with unknown parameters and randomly missing outputs, International Journal of Robust and Nonlinear Control 19 (18) (2009) 1976-1992. doi:10.1002/rnc.1390

[8] H. L. Mitchell, P. Houtekamer, Ensemble kalman filter configurations and their performance with the logistic map, Monthly Weather Review 137 (12) (2009) 4325-4343. doi : 10.1175/ 2009MWR2823.1.

[9] C.-C. Wu, G.-Y. Lien, J.-H. Chen, F. Zhang, Assimilation of tropical cyclone track and structure based on the ensemble kalman filter (enkf), Journal of the Atmospheric Sciences 67 (12) (2010) 3806-3822. doi:10.1175/2010JAS3444.1.

[10] T. Miyoshi, M. Kunii, The local ensemble transform kalman filter with the weather research and forecasting model: Experiments with real observations, Pure and applied geophysics 169 (3) (2012) 321-333. doi:10.1007/s00024-011-0373-4.

[11] S. Aoki, K. Amaya, M. Sahashi, T. Nakamura, Identification of gurson's material constants by using kalman filter, Computational Mechanics 19 (6) (1997) 501-506. doi:10.1007/ s004660050199.

[12] A. Corigliano, S. Mariani, B. Orsatti, Identification of gurson-tvergaard material model parameters via kalman filtering technique. i. theory, International journal of fracture 104 (4) (2000) 349-373. doi:10.1023/A:1007602106711.

[13] T. Nakamura, Y. Gu, Identification of elastic-plastic anisotropic parameters using instrumented indentation and inverse analysis, Mechanics of materials 39 (4) (2007) 340-356. doi: $10.1016 / j$.mechmat.2006.06.004

[14] T. Nakamura, Y. Liu, Determination of nonlinear properties of thermal sprayed ceramic coatings via inverse analysis, International journal of solids and structures 44 (6) (2007) 19902009. doi:10.1016/j.ijsolstr.2006.08.012

[15] G. Bolzon, R. Fedele, G. Maier, Parameter identification of a cohesive crack model by kalman filter, Computer Methods in Applied Mechanics and Engineering 191 (25) (2002) 2847-2871. doi:10.1016/S0045-7825(02)00223-2. 
[16] P. Vaddadi, T. Nakamura, R. P. Singh, Inverse analysis for transient moisture diffusion through fiber-reinforced composites, Acta materialia 51 (1) (2003) 177-193. doi:10.1016/ S1359-6454(02)00390-7.

[17] P. Vaddadi, T. Nakamura, R. P. Singh, Inverse analysis to determine hygrothermal properties in fiber reinforced composites, Journal of composite materials 41 (3) (2007) 309-334. doi : 10.1177/0021998306063372

[18] R. L. Plackett, Some theorems in least squares, JSTOR, 1950. URL http://www . jstor.org/stable/2332158

[19] G. Lindberg, A. Shokry, W. Reheman, I. Svensson, Determination of diffusion coefficients in bovine bone by means of conductivity measurement, International Journal of Experimental and Computational Biomechanics 2 (4) (2014) 324-342. doi:10.1504/IJECB.2014. 066086 .

[20] R. Courant, D. Hilbert, Methods of Mathematical Physics (Interscience, New York, 1953), Vol. 63.

[21] K. B. Petersen, M. S. Pedersen, The matrix cookbook, Technical University of Denmark (2008) 7-15.

URL WWW2 .imm.dtu.dk/pubdb/p.php?3274 